\title{
A NOTE ON AXISYMMETRIC SUPERCRITICAL CONING IN A POROUS MEDIUM
}

\author{
G. C. HOCKING ${ }^{凶 1}$ and H. ZHANG ${ }^{2}$ \\ (Received 8 January, 2014; revised 5 May, 2014; first published online 7 August 2014)
}

\begin{abstract}
The steady response of a fluid with two layers of different density in a porous medium is considered during extraction through a point sink. Supercritical withdrawal in which both layers are being withdrawn is investigated using a spectral method. We show that for each withdrawal rate, there is a single entry angle of the interface into the point sink. As the flow rate decreases the angle of entry steepens until it becomes almost vertical, at which point the method fails. This limit is shown to correspond to the upper bound on sub-critical (single-layer) flow.
\end{abstract}

2010 Mathematics subject classification: 0103.

Keywords and phrases: porous media, point sink, axisymmetric flow, coning.

\section{Introduction}

As water resources in dry climates are stretched further by population growth and rising salinity, it is of increasing importance to be able to manage water resources better. One source of water that is becoming more important internationally is underground aquifers, but as more pressure is placed on this resource the likelihood of salt water contamination increases. In this paper we consider withdrawal through a point sink from an unconfined aquifer containing two layers of different density which may be considered as a salt water layer beneath a fresh water layer. Similarly, increasing pressure on oil resources means more efficient withdrawal from oil reservoirs is becoming necessary. The problem of withdrawal from a layer of oil over water is mathematically identical to the problem of two layers of water of different density [8].

The unique part of the present work is that the withdrawal is simultaneously from both layers, that is, supercritical flow. Earlier work $[3,13,17]$ has concentrated on subcritical flows in which all of the extracted fluid comes from the layer adjacent to the

\footnotetext{
${ }^{1}$ Mathematics \& Statistics, Murdoch University, Perth, Australia; e-mail: G.Hocking@murdoch.edu.au.

${ }^{2}$ Griffith School of Engineering, Griffith University, Gold Coast Campus, QLD 4222, Australia; e-mail: h.zhang@griffith.edu.au.

(C) Australian Mathematical Society 2014, Serial-fee code 1446-1811/2014 \$16.00
} 


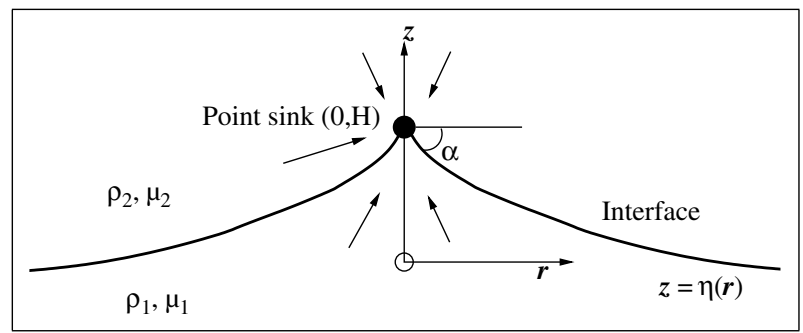

FigURE 1. Schematic of the flow into a point sink from two layers of different density-supercritical flow rate.

point sink. The critical flow rate is defined as the maximum rate at which only the fluid from the layer adjacent to the sink is withdrawn, and it is of great practical interest.

In order to consider this problem we treat the interface between the two fluids as a sharp interface where the two fluids do not mix. The case of withdrawal through a line sink (two-dimensional flow) began with the work of Muskat [15], and many scientists have studied critical withdrawal for various aquifer configurations; see, for example, $[2,6,11,14,17,18]$. The case of supercritical flow, in which both layers are withdrawn simultaneously through a sink, was considered by Yu [16] and Henderson et al. [7]. More recently, Hocking and Zhang [12] and Zhang et al. [19] found that supercritical withdrawal into a line sink was possible over a range of different flow rates for a given entry angle of the interface. This result is contrary to the analogous surface water, supercritical case $[4,9,10]$ in which solutions obtained with an integral equation technique showed that each flow rate into a line sink produced a unique angle of entry.

There is less work available on the three-dimensional (point sink) case. Blake and Kucera [3] and Lukas et al. [13] considered this problem, including flows with multiple sinks and sources in an attempt to modify the critical flow rate to allow higher withdrawal rates without breakthrough of salt water. Their work was restricted to the subcritical case. Forbes and Hocking [5] found a very limited range of supercritical solutions for surface water flow into a point sink.

In the present study, two homogeneous and isotropic aquifer layers separated by an interface of infinitesimal thickness are considered. A point sink is located in the upper layer and withdraws fluid at some constant rate (see Figure 1). The origin is situated at a distance $H$ directly beneath the sink at the level of the undisturbed interface. We seek supercritical solutions in which fluid from both layers is flowing out through the sink. Potential functions are chosen using appropriate eigenfunctions that satisfy the flow equations except at the interface itself. Orthogonality and an iterative scheme are exploited to compute series coefficients that satisfy the nonlinear boundary conditions. In each case we find that as the flow rate decreases the interface at the sink steepens until it becomes almost vertical, at which point the method fails. Thus, for each value of entry angle there is a single, unique withdrawal rate. As this withdrawal rate decreases the limiting flow approaches the single layer maximal coning flow. 


\section{Formulation}

2.1. Equations Consider a homogeneous and isotropic porous medium with permeability $\kappa$. Two fluids of different density and dynamic viscosity are separated by an interface of infinitesimal thickness into two homogeneous layers as seen in Figure 1. A point sink, $S$, of strength, $Q$, is located at a distance, $H$, above the origin. The fluids located beneath and above the interface are defined as fluid 1 and fluid 2, respectively.

We adopt a cylindrical polar coordinate system, and assume the flow to be axisymmetric so that the flow is independent of angle, $\theta$, and only depends on horizontal distance from the sink, $r$, and elevation above the undisturbed level of the interface, $z$. If $z=\eta(r)$ is the equation of the interface, suppose the region below the interface to have density $\rho_{1}$ and dynamic viscosity $\mu_{1}$ and the region above the interface to have density $\rho_{2}$ and dynamic viscosity $\mu_{2}$. The velocity potentials in each region in three-dimensional steady flow satisfy Darcy's law:

$$
\begin{array}{ll}
\Phi_{1}(r, z)=\frac{\kappa}{\mu_{1}}\left(p+\rho_{1} g z\right), & z<\eta(r), \\
\Phi_{2}(r, z)=\frac{\kappa}{\mu_{2}}\left(p+\rho_{2} g z\right), & z>\eta(r),
\end{array}
$$

where $\kappa$ is the intrinsic permeability, $p$ is the pressure at elevation $z$ and $g$ is gravitational acceleration. Matching the pressure across the interface between the two immiscible fluid regions gives a condition on the interface, $z=\eta(r)$, that

$$
\Phi_{1}-\gamma \Phi_{2}=K z \quad \text { where } \gamma=\frac{\mu_{2}}{\mu_{1}} \quad \text { and } \quad K=\frac{\kappa g\left(\rho_{1}-\rho_{2}\right)}{\mu_{1}} .
$$

In porous media flow into a line sink the interface cannot level off because of the logarithmic dependence of the sink potential and its relation to the interface condition. However, in the case of a point sink the potential is of the order of $0(1 / r)$ so that the surface converges to horizontal as $r \rightarrow \infty$.

The solutions we seek are those in which the interface is drawn up a distance $H$ to a point where it enters a point sink with an angle $\alpha$ (defined positive downward) to the horizontal, as shown in Figure 1. Since flux from each layer (see below) depends on the angle of entry, $\alpha$, the flux from the lower fluid is $Q((\pi / 2)-\alpha) / \pi$ and from the upper fluid it is $Q((\pi / 2)+\alpha) / \pi$. Fluid is withdrawn from both above and below the interface. The velocity potentials of the separate flow fields below and above the interface must satisfy Laplace's equation,

$$
\begin{array}{ll}
\nabla^{2} \Phi_{1}(r, z)=0, & z<\eta(r), \\
\nabla^{2} \Phi_{2}(r, z)=0, & z>\eta(r),
\end{array}
$$

and the velocity potentials must be point-sink like as the outlet is approached, so that

$$
\begin{aligned}
& \Phi_{1} \rightarrow \frac{\pi Q_{1}}{4 \pi[(\pi / 2)-\alpha]} \frac{1}{\left[r^{2}+(z-H)^{2}\right]^{1 / 2}} \quad \text { as }(r, z) \rightarrow(0, H), z \leq \eta(r), \\
& \Phi_{2} \rightarrow \frac{\pi Q_{2}}{4 \pi[(\pi / 2)+\alpha]} \frac{1}{\left[r^{2}+(z-H)^{2}\right]^{1 / 2}} \quad \text { as }(r, z) \rightarrow(0, H), z \geq \eta(r),
\end{aligned}
$$

where $Q_{1}$ and $Q_{2}$ are the respective total dimensional fluxes from within the two regions. There is a relationship between these two values which must hold if the 
dynamic condition on the interface is to be satisfied. Applying Darcy's law [1] to the streamline along the interface and noting that for steady flow there must be no pressure difference across the interface leads to

$$
\eta=\frac{1}{K}\left[\Phi_{1}(r, z)-\gamma \Phi_{2}(r, z)\right] \quad \text { on } z=\eta(r) .
$$

Defining the dimensionless variables

$$
z^{*}=\frac{z}{H}, \quad r^{*}=\frac{r}{H}, \quad \Phi_{1}^{*}=\frac{\Phi_{1}}{\gamma Q / 4 \pi H}, \quad \Phi_{2}^{*}=\frac{\Phi_{2}}{Q / 4 \pi H},
$$

the nondimensional form of the dynamic interface condition (2.6) becomes

and

$$
\eta^{*}=G\left(\Phi_{1}^{*}-\Phi_{2}^{*}\right) \quad \text { where } G=\frac{Q \gamma}{4 \pi K H^{2}}
$$

$$
\begin{aligned}
& \Phi_{1}^{*} \rightarrow \frac{1}{R^{*}} \quad \text { as }\left(r^{*}, z^{*}\right) \rightarrow(0,1), z^{*} \leq \eta^{*}\left(x^{*}\right), \\
& \Phi_{2}^{*} \rightarrow \frac{1}{R^{*}} \quad \text { as }\left(r^{*}, z^{*}\right) \rightarrow(0,1), z^{*} \geq \eta^{*}\left(r^{*}\right),
\end{aligned}
$$

while in the far field it is required that

$$
\Phi_{1}^{*} \rightarrow 0, \Phi_{2}^{*} \rightarrow 0 \quad \text { as } R^{*} \rightarrow \infty,
$$

where $R^{*}=\left[r^{* 2}+\left(z^{*}-1\right)^{2}\right]^{1 / 2}$. The asterisk denotes dimensionless variables, and henceforth will be dropped for simplicity. The quantity $G$ is, therefore, a measure of the flow strength. Another condition to be satisfied is that there be no flow across the interface, and this can be ensured by enforcing the condition that the flow is along the interface, that is,

$$
\begin{array}{ll}
\Phi_{1 z}=\eta^{\prime}(r) \Phi_{1 r} & \text { on } z=\eta(r), \\
\Phi_{2 z}=\eta^{\prime}(r) \Phi_{2 r} & \text { on } z=\eta(r) .
\end{array}
$$

2.2. Spectral method for supercritical withdrawal We now define potential functions for each region and an interface function that build the correct limiting behaviour both near the sink and in the far field, and then compute the corrections to these. The potential functions will provide the appropriate sink behaviour and satisfy Laplace's equation (2.4), and an appropriate choice is

$$
\begin{aligned}
& \Phi_{1}(r, z)=-\frac{1}{R}+\sum_{k=1}^{\infty} a_{k} e^{\lambda_{k}(z-1)} J_{0}\left(\lambda_{k} r\right), \quad z \leq \eta(r), \\
& \Phi_{2}(r, z)=-\frac{1}{R}+\sum_{k=1}^{\infty} b_{k} e^{-\lambda_{k} z} J_{0}\left(\lambda_{k} r\right), \quad z \geq \eta(r),
\end{aligned}
$$

where $a_{k}, b_{k}, k=1,2, \ldots$, are real coefficients to be computed, $\lambda_{k}, k=1,2, \ldots$, are appropriate eigenvalues, and $J_{0}$ is a Bessel function of the first kind. This form assumes that the series is valid over $0<r<L$ and the truncation point, $L$, is chosen to be large enough to provide converged solutions. The forms of $\Phi_{1}$ and $\Phi_{2}$ have the correct $1 / R$ behaviour in the far field, and so the correct boundary condition at $r=L$ is that the series terms make no additional contribution, so eigenvalues are chosen so that $J_{0}\left(\lambda_{k} L\right)=0$. This choice makes no difference to the computed solutions (see below). 
We also define

$$
\eta(r)=\eta_{0}(r)+\sum_{k=1}^{\infty} c_{k} J_{0}\left(\lambda_{k} r\right)
$$

where the $c_{k}, k=1,2, \ldots$, are to be determined, and $\eta_{0}(r)$ is carefully chosen to have the correct limiting behaviour as $r \rightarrow 0$ and $r \rightarrow \infty$ as

$$
\eta_{0}(r)=\frac{A r^{3}+(4-\tan \alpha) r^{2}+(6-4 \tan \alpha) r+1}{(r+1)^{4}} .
$$

Note that $\eta_{0}(0)=1, \eta_{0}^{\prime}(0)=-\tan \alpha$ and $\eta_{0} \rightarrow A / r$ as $r \rightarrow \infty$, which means the remaining terms in $\eta(r)$ will enable the use of orthogonality to satisfy the conditions. The constant $A$ is to be determined as an output of the method, but gives the behaviour of the potential (and hence interface) for large $r$.

At this point we might expect that we could substitute these series into (2.7) and (2.10), and solve for the series coefficients by Newton's method. However, this procedure proves to be highly ill-conditioned. An approach that works is to exploit the orthogonality of the eigenfunctions involved in each equation, producing an equation for each of the series coefficients. These equations are nonlinear, but can still be solved by an iterative method. Henceforth, we assume the value of $\gamma=1$, but the procedure if that is not the case is similar.

The equation (2.7) can be modified by invoking the orthogonality of the eigenfunctions to give an equation for the coefficients,

$$
c_{k}-\frac{2}{L^{2} J_{1}^{2}\left(\lambda_{k}\right)} \int_{0}^{L}\left[G\left(\Phi_{1}-\Phi_{2}\right)-\eta_{0}\right] r J_{0}\left(\lambda_{k} r\right) d r=0, \quad k=1,2,3, \ldots, N,
$$

where $L$ is the truncation point and $\lambda_{k}, k=1,2, \ldots$, are the appropriate eigenvalues of $J_{0}$ for this value of $L$. The other conditions (2.10) can be dealt with similarly, but one must be careful because the exponential terms (which make the orthogonality inviolate) cannot be ignored, and consequently we add and subtract terms so that the resulting equations for $a_{k}, b_{k}, k=1,2, \ldots$, are

$$
\begin{aligned}
& a_{k} \lambda_{k}-\frac{2}{L^{2} J_{1}^{2}\left(\lambda_{k}\right)} \int_{0}^{L} \eta_{0}^{\prime}(r) r J_{0}\left(\lambda_{k} r\right) d r \\
& \left.\quad-\frac{2}{L^{2} J_{1}^{2}\left(\lambda_{k}\right)} \int_{0}^{L} \sum_{j=1}^{\infty} a_{j} \lambda_{j}\left[\left(e^{\lambda_{j}(\eta-1)}+1\right) J_{0}\left(\lambda_{j} r\right)+e^{\lambda_{j}(\eta-1)} \eta^{\prime} J_{1}\left(\lambda_{j} r\right)\right)\right] r J_{0}\left(\lambda_{k} r\right) d r=0 \\
& b_{k} \lambda_{k}-\frac{2}{L^{2} J_{1}^{2}\left(\lambda_{k}\right)} \int_{0}^{L} \eta_{0}^{\prime}(r) r J_{0}\left(\lambda_{k} r\right) d r \\
& \quad+\frac{2}{L^{2} J_{1}^{2}\left(\lambda_{k}\right)} \int_{0}^{L} \sum_{j=1}^{\infty} b_{j} \lambda_{j}\left[\left(e^{-\lambda_{j} \eta}-1\right) J_{0}\left(\lambda_{j} r\right)-e^{-\lambda_{j} \eta} \eta^{\prime} J_{1}\left(\lambda_{j} r\right)\right] r J_{0}\left(\lambda_{k} r\right) d r=0
\end{aligned}
$$

for $k=1,2,3, \ldots$ The series can be truncated after $N$ terms, giving $3 N$ unknowns. 
If a "guess" is made for all series coefficients for $k=1,2, \ldots, N$, then (2.14)-(2.16) can be evaluated along the interface using Gaussian quadrature with as many points as we wish (here we use $M=15 N$ points to ensure accurate representation of each eigenfunction), and the error in each equation can be evaluated. Thus, we have $3 N$ equations for the $3 N$ unknown series coefficients. This was programmed into Octave and solved for the series coefficients (and hence the interface shape) using the fsolve algorithm. The convergence was tested as the computational window $L$ increased and the number of coefficients was increased, and it was found that with $L>40$, there was no discernible graphical difference in solutions (note that changing $L$ also changes the eigenvalues). Similarly, increasing the number of coefficients in the series beyond $N=200$ made no discernible difference to the graphical solutions (or to the $G$ flow values beyond three decimal places). Thus, solutions with $L=50$ and $N=250$ were used for most calculations. As the magnitude of the flow rate approached the limiting value from above, a greater number of coefficients in the series was required to provide suitable convergence.

It turns out that if entry angle $\alpha$ is an input, then flow rate $G$ is an output of the code, and so an extra equation is required to account for the extra unknown. The last coefficient of $\eta(r)$ was, therefore, neglected and replaced by $G$ in the equations. Also, the value of the constant $A$ was required as an output to provide a smooth solution near the truncation limit $L$, and so a simple extra equation that ensured a smooth continuation from $\eta_{N-1}$ to $\eta_{N}$ (using a one-term Taylor expansion) was added. Solutions for small values of $\alpha$ were computed using a starting guess with all coefficients being zero. The angle was then incremented using the smaller entry angle solutions as a starting guess, providing rapid convergence in each case.

\section{Results}

Results for several different values of $\alpha$ are shown in Figure 2. It is clear that as the flow rate decreases, the interface steepens at the entry point. The limiting case would be $\alpha=\pi / 2$, but the method began to struggle for values of $\alpha \geq 1.3$, as can be seen in Figure 2 for the case $\alpha=1.35$, where small wobbles can be seen on the interface. However, in spite of this difficulty, the values of $G$ for a given entry angle do seem to converge as $N$ is increased, so that with $N=250$ they are accurate to 3 decimal places. Figure 3 shows the flow rate computed at each angle. It is clear that as the entry angle $\alpha$ steepens, $G$ is approaching a value as the interface becomes vertical. Extrapolating the curve in Figure 3 to $\alpha=\pi / 2$ using the curve-fitting "trend line" facility in Excel gives $G \approx 0.167$. The work of Lukas et al. [13] gave a limiting single-layer flow value for $G \approx 0.163$ using an integral equation approach. (Note that their definition of $G$ is a factor of $4 \pi$ times bigger than that used here, and we have made the adjustment.) The consistency of these values suggests that the limiting single-layer flow is very close to this value. 


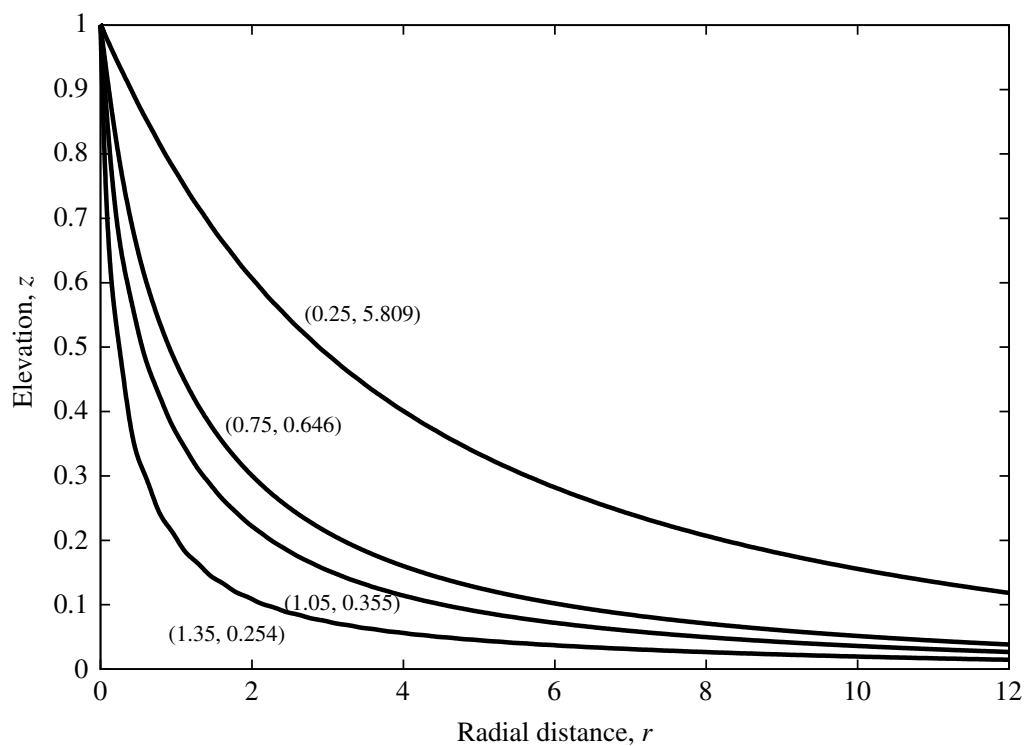

FigurE 2. Interface shapes with $(\alpha, G)=(0.25,5.809),(0.75,0.646),(1.05,0.355),(1.35,0.254)$. Note the steepening of the surface as the nondimensional flow rate, $G$, decreases.

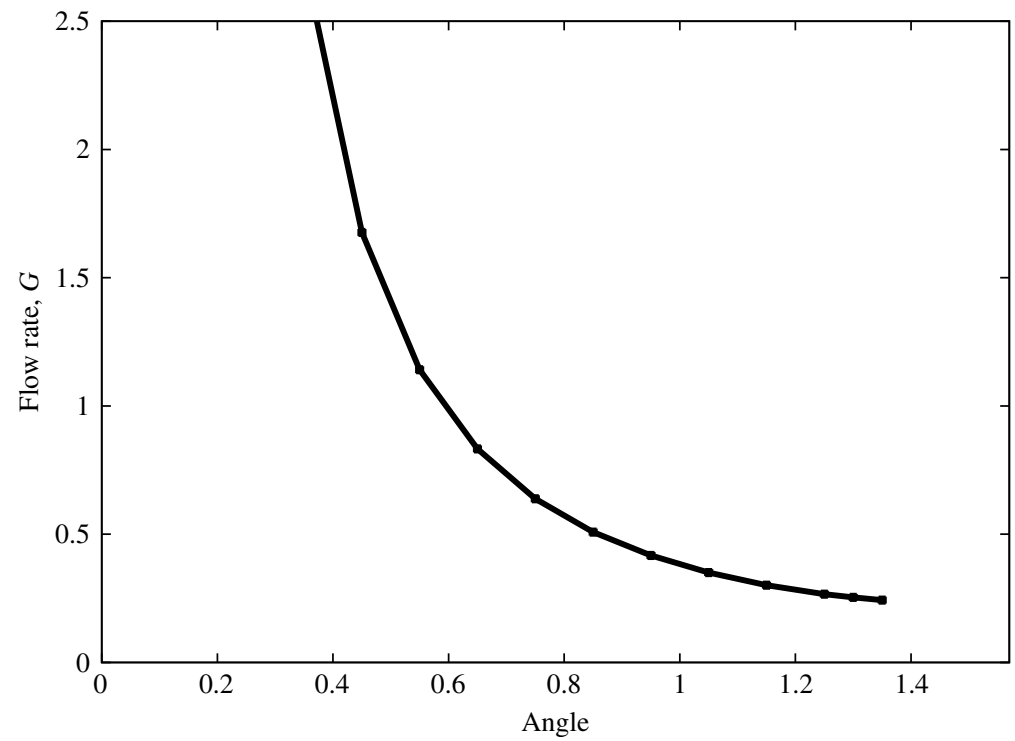

FIgURE 3. Flow rate value $G$ plotted against entry angle of the interface into the sink. The limiting value as $\alpha \rightarrow \pi / 2$ is about $G \approx 0.167$. 


\section{Conclusions}

The supercritical withdrawal through a point sink from two layers of fluid of different density in a homogeneous, isotropic two-dimensional aquifer was investigated. We used a spectral method to compute the interface shapes for the supercritical case in which both fluids, and consequently the interface, are drawn directly into the point sink. The difficulty is that this leads to a singularity on the interface itself. The method was able to deal with this singularity and is efficient and relatively easy to implement, but was unable to compute solutions right up to an angle of $\alpha \approx \pi / 2$.

As the value of entry angle, $\alpha$, steepens, the value of $G$ decreases, seeming to close in on a value near to $G=0.167$, very close to the limiting single-layer flow solutions $(G=0.163,[13])$.

The results raise a clear point of difference between the withdrawal through a point sink and the earlier work for a line sink $[12,17]$, in which there were multiple flow rate values valid for each entry angle of the interface. As the entry angle steepened the range of values of flow rate narrowed, seeming to approach a single value as $\alpha \rightarrow \pi / 2$. The reason for this is not clear, but it would seem that it is due in part to the fact that the logarithmic nature of the line sink potential does not allow the interface to approach zero as one moves away from the sink. This makes the balance to obtain a level interface in the far field very difficult and in some situations impossible, since the elevation of the interface is proportional to $\Phi$. On the other hand, $\Phi \rightarrow 1 / R$ for the point sink, simplifying the behaviour considerably as the surface elevation approaches a horizontal level in all cases.

The spectral method has provided an effective method for the computation of a supercritical, two-layer flow in three dimensions (point sink), in which there is a singularity on the interface, and provides an effective method to find similar solutions in other geometries.

\section{References}

[1] J. Bear, Dynamics of fluids in porous media (McGraw-Hill, New York, 1972).

[2] J. Bear and G. Dagan, "Some exact solutions of interface problems by means of the hodograph method", J. Geophys. Res. 69 (1964) 1563-1572; doi:10.1029/JZ069i008p01563.

[3] J. R. Blake and A. Kucera, "Coning in oil reservoirs", Math. Sci. 13 (1988) 36-47.

[4] L. K. Forbes and G. C. Hocking, "Withdrawal from a two-layer inviscid fluid in a duct", J. Fluid Mech. 361 (1998) 275-296; doi:10.1017/S0022112098008805.

[5] L. K. Forbes and G. C. Hocking, "On the computation of steady axi-symmetric withdrawal from a two-layer fluid”, Comput. Fluids 32 (2003) 385-401; doi:10.1016/S0045-7930(01)00085-8.

[6] F. M. Giger, "Analytic 2-D models of water cresting before breakthrough for horizontal wells", SPE Res. Engnrg. (1989) 409-416.

[7] N. Henderson, E. Flores, M. Sampaio, L. Freitas and G. M. Platt, "Supercritical fluid flow in porous media: modeling and simulation”, Chem. Eng. Sci. 60 (2005) 1797-1808; doi:10.1016/j.ces.2004.11.012.

[8] E. J. Hinch, "The recovery of oil from underground reservoirs", J. Physico-Chemical Hydrodynamics 6 (1985) 601-622. 
[9] G. C. Hocking, "Supercritical withdrawal from a two-layer fluid through a line sink", J. Fluid Mech. 297 (1995) 37-47; doi:10.1017/S0022112095002990.

[10] G. C. Hocking and L. K. Forbes, "Supercritical withdrawal from a two-layer fluid through a line sink if the lower layer is of finite depth", J. Fluid Mech. 428 (2001) 333-348; doi:10.1017/S0022112000002780.

[11] G. C. Hocking and H. Zhang, "A note on withdrawal from a two-layer fluid through a line sink in a porous medium", ANZIAM J. 50 (2008) 101-110; doi:10.1017/S144618110800028X.

[12] G. C. Hocking and H. Zhang, "Coning during withdrawal from two fluids of different density in a porous medium", J. Eng. Math. 65 (2009) 101-109; doi:10.1007/s10665-009-9267-1.

[13] S. K. Lukas, J. R. Blake and A. Kucera, "A boundary-integral method applied to water coning in oil reservoirs", J. Aust. Math. Soc. Ser. B 32 (1991) 261-283; doi:10.1017/S0334270000006858.

[14] J. F. McCarthy, "Gas and water cresting towards horizontal wells", J. Aust. Math. Soc. Ser. B 35 (1993) 174-197; doi:10.1017/S0334270000009115.

[15] M. Muskat and R. D. Wyckoff, "An approximate theory of water coning in oil production", Trans. AIME (Petroleum Development Technologies) 114 (1935) 144-163.

[16] D. Yu, K. Jackson and T. C. Harmon, "Dispersion and diffusion in porous media under supercritical conditions", Chem. Eng. Sci. 54 (1999) 357-367; doi:10.1016/S0009-2509(98)00271-1.

[17] H. Zhang and G. C. Hocking, "Axisymmetric flow in an oil reservoir of finite depth caused by a point sink above an oil-water interface", J. Eng. Math. 32 (1997) 365-376; doi: $10.1017 /$ S0334270000008845.

[18] H. Zhang, G. C. Hocking and D. A. Barry, "An analytical solution for critical withdrawal of layered fluid through a line sink in a porous medium", J. Aust. Math. Soc. Ser. B 39 (1997) 271-279; doi:10.1017/S0334270000008845.

[19] H. Zhang, G. C. Hocking and B. Seymour, "Critical and supercritical withdrawal from a two-layer fluid through a line sink in a partially bounded aquifer", Adv. Water Resour. 32 (2009) 1703-1710; doi:10.1016/j.advwatres.2009.09.002. 